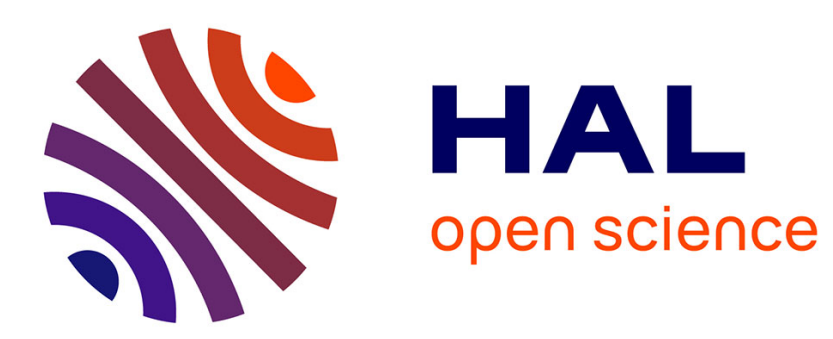

\title{
LE DÉTACHEMENT EST-IL UNE PROPRIÉTÉ BASIQUEDE LA PRÉDICATION SECONDE?
}

\author{
Eva Havu, Michel Pierrard
}

\section{To cite this version:}

Eva Havu, Michel Pierrard. LE DÉTACHEMENT EST-IL UNE PROPRIÉTÉ BASIQUEDE LA PRÉDICATION SECONDE?. L'information grammaticale, 2006, 109, pp.20-26. halshs-00354002

\section{HAL Id: halshs-00354002 \\ https://shs.hal.science/halshs-00354002}

Submitted on 2 Feb 2009

HAL is a multi-disciplinary open access archive for the deposit and dissemination of scientific research documents, whether they are published or not. The documents may come from teaching and research institutions in France or abroad, or from public or private research centers.
L'archive ouverte pluridisciplinaire HAL, est destinée au dépôt et à la diffusion de documents scientifiques de niveau recherche, publiés ou non, émanant des établissements d'enseignement et de recherche français ou étrangers, des laboratoires publics ou privés. 


\title{
Le détachement est-il une propriété basique de la prédication seconde?
}

\author{
Eva HAVU \& Michel PIERRARD \\ Université d'Helsinki, Finlande - Vrije Universiteit Brussel, Belgique
}

Les études sur le détachement (DÉT) lient souvent ce type de construction au fonctionnement de la prédication seconde (PRÉD2): la construction détachée "introduit une nouvelle structure prédicative (...), vient s'ajouter toujours à une prédication première dans une relation plus ou moins lâche" (Combettes $1998: 12-13)$. Les deux notions sont également associées pour définir d'autres fonctions ; Forsgren (2000: 34) ou Neveu (1998: 69) ${ }^{1}$ par exemple, les combinent dans la définition de l'apposition. De fait, un survol rapide de quelques constructions de type PRÉD2 semble confirmer le lien étroit entre la prédication seconde et le détachement :

(1) a) Paris, ville des sciences, est la capitale de la France

b) Professeur de français, il passe toutes ses vacances en France.

c) Furieux, Jean est parti.

d) Regardant à sa gauche, il vit le facteur qui sortait de sa maison.

e) Reposé et de bonne humeur, il est allé travailler.

La prédication seconde est globalement caractérisée comme "un type de séquence qui, malgré son statut syntaxiquement intégré, exprime sémantiquement un contenu phrastique à l'intérieur même de la phrase" (Cadiot \& Furukawa $2000: 3$ ). Nous confronterons cette définition aux critères proposés pour définir le détachement (en particulier dans Fradin 1988; 1990, Combettes 1998, Neveu 2003) afin d'évaluer dans quelle mesure ces deux notions recouvrent des propriétés identiques et sous quelles conditions elles peuvent se combiner.

Définir le détachement est une entreprise particulièrement complexe : "la notion de détachement est tout à la fois surexploitée et fort peu lisible" (Neveu 2003 : 7). A la base, elle est censée caractériser une "discontinuité dans la linéarité du discours" (Ibid.); des propriétés diverses lui sont attribuées : pour Dupont (1985 : 14), citant le Grand Larousse de la Langue Française, le 'détachement' implique "une individualisation phonétique qui s'accompagne ou non d'un déplacement". Par ailleurs, le complément détaché sera, d'un point de vue syntaxo-sémantique "une annexe de phrase, un supplément de proposition" (Ibid. : 16). Fradin (1990:24) y rajoute la 'Condition du Rapport Obligé' (CRO) : "tout élément détaché doit entrer dans une relation sémantique $r$ avec (un élément de) l'énoncé auquel il est incident". Ainsi, selon le trait mis en exergue, on distinguera trois saisies basiques, fondant successivement le détachement (1) sur une relation sémantique, (2) sur la saillance de traits formels et enfin (3) sur un fonctionnement syntaxo-sémantique spécifique. Nous examinerons les différents cas de figure pour évaluer en quelle mesure ils favorisent une convergence avec les propriétés de PRÉD2.

\section{DÉT en tant que relation sémantique}

La caractérisation du détachement par une relation sémantique trouve sa forme canonique dans un rapport référentiel et/ ou fonctionnel entre un élément de rappel dans le segment détaché et un

\footnotetext{
1 “Seul semble pouvoir être reçu comme critère fiable de l'apposition, c'est-à-dire extensible à tous les types de constructions, celui de la prédication seconde marquée par le détachement" (1998:69).
} 
élément de la prédication centrale ${ }^{2}$. Le détachement évoquera alors en particulier les structures disloquées, généralement représentées par les énoncés suivants :

(2) a) Le facteur, personne ne l'attendait à cette heure. (Fradin 1990:3)

b) Il n'est pas venu, lui. (Prévost $2003: 104)$

c) Paul, sa voiture, elle roule à $200 \mathrm{~km} / \mathrm{h}$. (Prévost $2003: 104$ )

d) Un homme, ça doit être fort (Furukawa 2003:128) / La mer, c'est beau (Willems $1998: 595$ )

e) Satisfait de son travail, il ne l'était guère. (Furukawa $2003: 139$ )

Dans ce cas, les propriétés spécifiques à prendre en considération pour rendre compte de cette relation sémantique entre le segment disloqué et l'élément de rappel seront les suivantes (cf. Lambrecht 2001, Prévost $2003: 105)$ :

P1 le caractère nécessairement référentiel de l'élément détaché (ou au moins son aptitude à être construit comme référent discursif) ;

P2 l'alternance possible de la position détachée (extra-propositionnelle) avec une position intégrée (intra-propositionnelle);

P3 la présence d'une coindexation pronominale, en particulier à travers une relation GN-clitique.

\subsection{Restrictions générales}

En gros, l'ensemble des énoncés $2 \mathrm{a}$-e est censé répondre aux trois critères avancés, même si $2 \mathrm{~d}$ et 2e présentent certaines spécificités par rapport au trait $\mathrm{P} 3$ et que le segment détaché dans (2e) ne répond pas de prime abord aux conditions du trait P1 (cf. Furukawa 2003, Prévost 2003). De fait, des limitations plus générales sont d'application en ce qui concerne la présence nécessaire d'un élément de rappel (1.1.1.) et surtout par rapport au caractère référentiel de l'élément détaché (1.1.2.)

\subsubsection{La coindexation pronominale :}

Les constructions topicalisées (3a) sont généralement considérées comme des constructions détachées (cf. Fradin 1990 : 12). Or, elles n'ont pas d'élément de rappel et par conséquent il n’y a pas de coindexation pronominale. C'est également le cas des 'constructions détachées sans rappel' (Fradin $1988: 26$, ex. 3b) :

(3) a) De cette histoire, il se souviendra longtemps/ La politique, vous connaissez ?

b) L'armée, j'ai déjà donné

Dans ces cas-là, Fradin parle d'une relation sémantique 'fonctionnelle' entre l'élément détaché et une position d'argument vide.

\subsubsection{Le caractère nécessairement référentiel de l'élément détaché :}

Cette 'référentialité' doit également être relativisée. Ainsi, pour Furukawa (2003 : 130-146), les adjectifs et les noms précédés d'un article indéfini sont effectivement des éléments non référentiels (non identifiés référentiellement) mais leur topicalisation serait métalinguistique. Grâce à leur reprise par un pronom (cf. malade, il l'est), ils obtiendraient un référent intralinguistique et non extralinguistique comme dans le cas des noms définis. Une autre façon de

\footnotetext{
2 "Quand l'élément incident comporte un élément de rappel, $r$ prend le visage d'une relation référentielle et/ ou fonctionnelle $[\ldots]$. Quand un tel élément est absent, $r$ est une relation interprétative qui peut être soit prédicative $[\ldots]$, soit de repérage [...], soit d'inclusion [...], soit encore classificatoire [...]" (Fradin 1990:24)
} 
résoudre le problème est de lier leur référentialité à leur incidence à une catégorie nominale ou propositionnelle au sein d'une unité supérieure : l'élément détaché sera alors "le développement d'une catégorie syntaxique majeure, c'est-à-dire X" ou encore une phrase (S ou S')", qui s'interprète soit "par rapport au contenu de la phrase à laquelle il est incident" (argument dans l'évenance rapportée par le $\mathrm{S}$ incident ou circonstant), soit "indépendamment de cette évenance (e.g. par rapport à l'acte d'énonciation)" (Fradin 1990 : 11).

\subsection{Propriétés sémantiques de DÉT et PRÉD2}

En quelle mesure les tours classiquement regroupés sous l'étiquette de PRÉD2 peuvent-ils être caractérisés par les propriétés du détachement telles qu'elles ont été définies ci-dessus?

\subsubsection{Le caractère nécessairement référentiel de l'élément détaché.}

Si le groupe disloqué est lui-même référentiel (4a : propriété P1 ; cf. Combettes 1998 : 14), les prédications secondes détachées ne le sont généralement pas (4b), sauf les constructions appositives 'identifiantes' (Forsgren 1986 : 607) (4c), qui peuvent effectivement être considérées comme référentielles. Paradoxalement toutefois, Combettes (1998:16) ne considère justement pas ces tours comme des constructions détachées "[...] l'on ne peut confondre les syntagmes nominaux sans déterminant et les syntagmes avec déterminant [...], ces derniers ne devant pas être classés parmi les $\mathrm{CD}$ en raison de leurs caractéristiques syntaxiques et référentielles" :

(4) a) Marie, elle est fort malade.

b) Imprudente, Marie est tombée malade.

c) Marie, la sœur aînée de notre collègue, est fort malade.

A un niveau plus global, le trait de 'référentialité' se heurte à un obstacle de fond dans le cas des PRÉD2 puisqu'il implique que les prédicats seconds constituent des N. Or, ils représentent au contraire un apport prédicatif greffé sur un $\mathrm{N}$ de la prédication régissante :

(5) a) Furieux, l'homme est sorti.

$[<$ L'homme (est) furieux]

b) Regardant à sa gauche, il vit le facteur qui sortait de sa maison.

$[<$ Il regarde à sa gauche]

c) Reposé et de bonne humeur, il est allé travailler.

[ $<$ Il est reposé et de bonne humeur]

La notion de 'catégorie syntaxique majeure' (cf. Fradin ci-dessus) pose également problème dans la mesure où la PRÉD2 n'est jamais un argument de l'évenance (cf. Blasco-Dulbecco \& Caddéo 2001 : 130) ; d'ailleurs une PRÉD2 ne peut jamais entrer dans une position argumentale ouverte, ce qui est par contre parfaitement possible dans le cas des 'constructions détachées sans rappel' comme (3a) :

(3) a) De cette histoire, il se souviendra longtemps.

['de cette histoire' = complément prépositionnel du prédicat principal]

En effet, l'adjectif furieux dans (5a) n'est pas dans un rapport d'incidence au sein d'une unité supérieure (syntagme nominal ou verbal), et ceci ni envers le prédicat, ni envers un de ses arguments, contrairement au même adjectif dans (5a'), qui est incidenciel à homme au sein du SN l'homme furieux :

(5) a) Furieux, l'homme est sorti.

a') L'homme furieux a quitté la pièce. 
Une analyse similaire sera appliquée au cas particulier du détachement adjectival sous (2e); l'élément détaché y participe effectivement à la PRÉD1, et il y est d'ailleurs représenté par un clitique, ce qui n'est jamais le cas des PRÉD2. Il n'y a donc pas dans (2e) constitution d'une prédication seconde :

(2) e) Satisfait de son travail, il ne l'était guère. (Furukawa $2003: 139$ )

\subsubsection{L'alternance des positions}

L'alternance entre les positions détachée et intégrée (propriété P2), qui ne pose aucun problème dans le cas des disloquées (cf. 6a ; 2a ; 3a), est impossible dans les tours (1a-e). Qui plus est, cette impossibilité est une propriété définitoire des PRÉD2 considérées (sauf encore une fois dans le cas des appositions 'identifiantes'(4c)) :

(6) a) Paul, il a pris le train ce matin

(2) Paul a pris le train ce matin.
Le facteur, personne ne l'attendait à cette heure.

= personne n'attendait le facteur à cette heure

(3) a) De cette histoire, il se souviendra longtemps.

(4) c) Marie, la sæur aînée de notre collègue, est fort malade.

= La sœur aînée de notre collègue est fort malade.

(1) a) Paris, ville des sciences, est la capitale de la France

*ville des sciences est la capitale de la France.

b) Professeur de français, il passe toutes ses vacances en France.

* Professeur de français passe toutes ses vacances en France.

c) Furieux, l'homme est sorti.

*Furieux est sorti.

d) Regardant à sa gauche, il vit le facteur qui sortait de sa maison. *Regardant à sa gauche vit le facteur qui sortait de sa maison.

e) Reposé et de bonne humeur, il est allé travailler. *Reposé et de bonne humeur est allé travailler.

En outre, la PRÉD2 à l'initiale peut avoir une valeur circonstancielle, ce qui est lié au fait qu'il y a greffe d'un rapport prédicatif second sur la prédication centrale (6b), alors que cela n'est jamais le cas pour les disloquées, qui explicitent un topique (Prévost $2003: 119$ ), et dont le fonctionnement sémantique est de type référentiel (6a') :

(6) b) Furieux, Paul a refusé de prendre le train ce matin.

$=$ comme il était furieux, ...

a') Paul, il a pris le train ce matin

$\neq$ Comme Paul a pris le train, il ....

Ces deux observations corroborent l'intuition que les énoncés (6a) et (6b) supposent des orientations fondamentalement différentes (cf. aussi à ce propos Neveu 2003: 12). Les dislocations de type (6a) manifestent une rupture de la continuité phrastique, une sortie du cadre propositionnel, tandis que les prédications secondes de type (6b) soulignent au contraire une intégration de prédications, donc une entrée dans le cadre propositionnel.

\subsubsection{La coindexation pronominale.}

La coindexation au moyen d'un clitique (propriété P3) n'est généralement pas de mise en PRÉD2 du fait que l'élément prédicatif n'est pas un argument direct du verbe régissant : 
(6) a) $\quad \operatorname{Paul}_{i}, \mathrm{il}_{\mathrm{i}}$ a pris le train ce matin

(2) a) Le facteur $i$, personne ne $l_{i}$ 'attendait à cette heure.

(1) c) ??Furieux ${ }_{i}, \mathrm{il}_{\mathrm{i}}$ est sorti.

d) ??Regardant ${ }_{i}$ à sa gauche, $\mathrm{il}_{\mathrm{i}}$ vit le facteur qui sortait de sa maison.

e) ??Reposé $e_{i}$ et de bonne humeur, $\mathrm{il}_{\mathrm{i}}$ est allé travailler.

De fait, dans 1c-e, le rapport n'est pas un rapport de coindexation de deux N, dont l'un occupe une place argumentale auprès du prédicat régissant, mais un rapport thème - prédicat. C'est ce type de rapport fondamentalement différent qui explique pourquoi les 'attributs de l'objet' ne permettent pas la coindexation (7b), contrairement aux attributs du sujet classiques qui sont considérés comme un argument direct de la copule être (7a) :

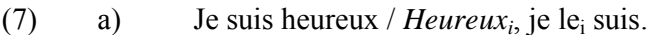

b) Il a acheté sa voiture neuve / $* N e u v e_{i}$, il $l_{i}$ 'a acheté sa voiture

La situation paraît différente dans les énoncés 1a'-b et 8 , où le parallélisme avec les constructions disloquées semble plus forte

(1) a') Ville des sciences $_{i}$, elle $_{i}$ est la capitale de la France.

b) Professeur de français ${ }_{i}$, $\mathrm{il}_{\mathrm{i}}$ passe toutes ses vacances en France.

(8) Petit Lillois de Paris ${ }_{i}$, rien ne $\mathrm{me}_{\mathrm{i}}$ frappait d'avantage que les symboles de nos gloires (Grevisse $1993: 549 \S 353$

L'identité n'est toutefois qu'une apparence. Dans le cas des disloquées, le clitique est incontournable puisqu'il doit pouvoir être remplacé par l'élément disloqué (6a-a'). Dans le cas des PRÉD2, il peut être remplacé par un N quelconque mais pas par l'élément détaché (1b-b') :

(6) a) Paul, il a pris le train ce matin

a') *Paul, mon frère a pris le train ce matin ( $\neq$ structure disloquée)

a") Paul a pris le train ce matin

(1) b) Professeur de français, il passe toutes ses vacances en France.

b') Professeur de français, Paul/ mon frère/ cet homme passe toutes ses vacances en France.

b") *Professeur de français passe toutes ses vacances en France.

Le rapport entre le clitique et le prédicat second n'implique donc pas la coindexation pronominale caractérisant les disloquées. La différence fondamentale entre les deux types de rapports instaurés apparaît encore avec les structures appositives identifiantes (9a) où il est impossible de placer le SN identifiant en position post $\mathrm{N}(9 \mathrm{c})$, position spécifique aux structures en apposition. Le clitique impose dans ce cas-là automatiquement une lecture disloquée $(9 \mathrm{~b})$, tandis que le pronom tonique permet parfaitement une lecture de type appositive (9d). Là où la confusion entre les deux opérations pourrait s'instaurer, le caractère clitique ou non clitique du pronom joue un rôle déterminant. :

(9) a) Paul, le frère aîné de notre collègue, est fort malade.

b) Le frère aîné de notre collègue, il est fort malade.

c) *Il, le frère aîné de notre collègue, est fort malade.

d) Lui, le frère aîné de notre collègue, est fort malade.

Ceci n'est évidemment pas le cas dans d'autres tours prédicatifs seconds où un clitique peut parfaitement être le thème de PRÉD2

(9) e) Il marchait, seul, rêveur, captif de vagues sombres. (Grevisse $1993: 549$ § 352c). 
L'addition de ces arguments tend à suggérer qu'il faut distinguer radicalement les relations sémantiques référentielles et fonctionnelles, propres aux disloquées et aux topicalisées, des relations sémantiques prédicatives, constitutives de PRÉD2, et qu'en conséquence, la CRO, qui prétend intégrer des relations référentielles et prédicatives en une seule classe, paraît peu pertinente pour fonder le rapport entre détachement et prédication seconde.

\section{DÉT et indices formels de séparation}

Une deuxième manière d'appréhender le détachement est de le définir simplement comme une séparation formelle. Ce sont alors les indices formels de discontinuité qui seront considérés comme pertinents. Ceux-ci pourront se concrétiser sur deux plans :

P4 une séparation prosodique et graphique (intonation, prosodie, pause, ponctuation), qui distingue le segment détaché du reste de l'énoncé (Dupont 1985, Fradin 1988, 1990 ; Lambrecht 2001 ; Neveu 2003);

P5 une position syntaxique "périphérique" qui situe l'élément détaché en position syntaxiquement extra-propositionnelle/ séparée, peu intégrée à la structure phrastique canonique (Combettes 1998 ; Fradin 1988 ; 1990 ; Neveu 2003 ; Prévost 2003).

Les PRÉD2 peuvent-elles être purement et simplement définies comme des structures formellement séparées? Quelle est dans ce cadre la valeur opératoire de 'l'identification phonétique' ou de la position 'périphérique'?

\subsection{Restrictions générales}

'L'identification phonétique' du détachement est généralement réalisée en prenant en considération les pauses ou l'intonation. En quelle mesure ces marques formelles sont-elles véritablement pertinentes pour identifier les constructions détachées?

- La pause : les constructions détachées sans pause intonatoire sont possibles en langue parlée (Fradin 1990 : 7). Blanche-Benveniste \& Caddéo (2000 : 66) confirment que "La pause n'est pas un critère sûr pour définir l'apposition [...] Il arrive qu'elle soit simplement absente" :

(10) a) Mon père il a tout réparé tout seul (Fradin 1990: 7).

b) Adolescente vous lisiez beaucoup (Blanche-Benveniste \& Caddéo $2000: 66$ ).

Morel (1992) soulignait déjà que les mesures effectuées révèlent que les constituants thématiques disloqués avant le verbe ne sont pas suivis d'une pause. Par contre, l'élément disloqué après le verbe serait précédé d'une pause moins longue que celle précédant un élément apposé (cf. Blasco-Dulbecco 1999 : 45).

- L'intonation : Mertens (1990) affirme que la mélodie distinctive entre la construction verbale et l'élément disloqué serait plus pertinente que le critère de la pause. Mais pour Prévost (2003: 106) toutefois, la prosodie spécifique n'affecterait que l'élément détaché à gauche. La dimension intonatoire ne marquerait donc pas toutes les positions détachées ou en tous cas pas de la même manière. Ainsi, l'intonation sur le segment détaché n'est pas identique en (11a) et (11b) :

(11) a) Enfant $\uparrow$, Octave avait toujours très faim.

b) Octave avait toujours très faim, enfant $\downarrow$. 
Cette dernière position semble confirmée par d'autres travaux. Blasco-Dulbecco (1999: 45) soulignait déjà que la caractéristique de l'intonation haute de l'élément disloqué avant le verbe (donc à gauche) se retrouve dans l'analyse intonative menée par l'Institut de phonétique d'Aixen-Provence : l'élément disloqué recevrait « un intonème progrédient noté, alors que le reste de la $\mathrm{CV}$ recevrait un intonème conclusif marqué ». L'élément disloqué à droite offre par contre une mélodie plate, avec un ton montant final, une intonation qui serait comparable à l'incise (Morel $1992: 31)$.

Si les divers phénomènes phonétiques apparaissent bien dans un grand nombre d'occurrences des structures détachées, aucun ne semble pouvoir identifier de manière univoque les différents types de détachement et a fortiori les PRÉD2, où certains tours (12a-b) semblent même insensibles à l'identification phonétique :

(12) a) Il a le nez rouge.

b) Il estime la solution excellente.

c) Il est mort jeune.

d) Il a acheté sa voiture neuve.

Et lorsqu'une marque d'identification apparaît en PRÉD2 (12c-d), elle semble contraire aux tendances générales constatées pour le détachement : alors que c'est essentiellement la pause qui caractériserait le détachement à droite, $12 \mathrm{c}$-d se singulariseraient plutôt par une intonation particulière.

\subsection{Indices formels et PRÉD2}

La position périphérique implique pour sa part que l'élément détaché se trouve en position extérieure par rapport à la structure phrastique canonique. L'approche semble une fois de plus liée à la notion de dislocation, 'éjectant' un élément hors de la structure canonique de l'énoncé. Or, cela est loin d'être toujours le cas pour les PRÉD2 :

(1) a) Paris, ville des sciences, est la capitale de la France

(13) a) Mon frère, professeur de français, passe toutes ses vacances en France.

b) Jean, furieux, a refusé de partir.

c) L'homme, regardant à sa gauche, vit le facteur qui sortait de sa maison.

Par ailleurs, identification phonétique et position périphérique ne vont pas nécessairement de pair, comme le soulignent les énoncés 14a-c. Dans (14a), Fradin affirme qu'il n'y a pas d'identification phonétique ; celle-ci ne s'imposerait pas puisque la folle est clairement extraposée à droite de l'énoncé elle n'est pas revenue. Dans (14b) au contraire, l'identification phonétique est indispensable dans la mesure où l'adjectif est en position d'intégration classique au sein du GN. Enfin, dans (14c), la saturation fonctionnelle de être [+SUJET ; +LIEU] permet d'interpréter le segment la mère Raymonde comme extra-propositionnel malgré l'absence d'identification phonétique et une position non périphérique pour un énoncé interrogatif. D’une manière générale, il faut souligner la remarque de Fradin: "Si d'autres marques [pour indiquer qu'un constituant n'est pas sur le même plan que ses voisins] sont disponibles, le recours à l'intonation devient dispensable" (1990:9) :

(14) a) Non non non elle est pas revenue la folle mais on avait pas fini (Fradin $1990: 7$ )

[- individualisation phonétique ; + position périphérique]

b) Le client, furieux, a quitté le magasin.

[+ individualisation phonétique ; - position périphérique $]$

c) Où elle est la mère Raymonde ? (Fradin $1990: 8$ ) / Où est la mère Raymonde ? 
[- individualisation phonétique ; - position périphérique]

L'identification phonétique et la position périphérique peuvent en outre apparaître dans des structures prédicatives autonomes qui ne paraissent guère "exprimer sémantiquement un contenu phrastique à l'intérieur même de la phrase", donc une PRÉD2 :

(15) a) un homme est venu : le facteur (Dupont,...)

[un homme est venu : (c'était) le facteur]

b) Dès qu'il fut parti, Anna alla rejoindre ses sœurs. (Fradin $1990: 4$ )

Si les différentes traces et marques de séparation formelle, qui apparaissent dans les constructions détachées, touchent aussi les divers types de PRÉD2, elles ne permettent aucunement de les définir de manière univoque. Les PRÉD2 ne peuvent donc pas être purement et simplement définies comme des structures formellement séparées.

\section{DET et rôle syntaxo-sémantique accessoire}

La séparation de la forme détachée par rapport à la prédication principale peut induire une assimilation du détachement à l'expression d'un apport accessoire, ce qui se retrouve dans une sixième propriété définitoire, souvent avancée pour caractériser la construction :

P6 le caractère sémantiquement non déterminant pour l'interprétation de PRÉD1 et donc supprimable de DÉT : la construction détachée se trouve en surplus des éléments dont l'agencement suffit à constituer un énoncé complet ; c'est souvent "une parenthèse, une sorte d'incise explicative" qui s'ajoute à la prédication première (Combettes 1998: 12). En conséquence, le détachement peut être le produit d'une topicalisation mais ne peut pas introduire un élément nouveau (cf. Blasco-Dulbecco 1999 : 60-63) ${ }^{3}$.

Est-ce cette notion d' "apport secondaire" que le détachement partage avec la prédication seconde ? Cela semble effectivement s'appliquer à divers cas de PRÉD2 :

(16) a) Paul, l'ami de ma sœur, est parti à Paris hier soir / Paul est parti à Paris hier soir.

b) Furieux, l'homme a quitté la pièce / L'homme a quitté la pièce.

En y regardant toutefois de plus près, il faut constater que le critère reste insuffisant à la fois pour interpréter tous les cas de détachement et pour appréhender les caractéristiques de la PRÉD2 .

\subsection{Restrictions générales}

La notion d'apport sémantique accessoire ne permet pas de traiter le cas des topicalisés (3a-b) ou des 'détachées sans élément de rappel' (3c), qui sont agrammaticales sans l'élément détaché :

(3) a) De cette histoire, il se souviendra longtemps/ ?? Il se souviendra longtemps.

b) La politique, vous connaissez ? / ?? Vous connaissez ?

c) L'armée, j’ai déjà donné / ?? J'ai déjà donné.

\footnotetext{
${ }^{3}$ On peut trouver des exceptions pour diverses raisons stylistiques et psychologisantes. Blasco-Dulbecco (1999 : 63) donne un exemple où l'élément disloqué ne reprend pas un thème, mais serait utilisée pour poser d'emblée le thème central du poème : Mallarmé commence le poème Aprés-midi d’un faune par le vers : Ces nymphes, je les veux perpétuer.
} 
La seule interprétation possible de la notion d'accessoire se situera alors sur un plan informationnel : ainsi, la prédiction sera avancée que l'élément détaché ne pourra introduire un élément nouveau (cf. Blasco-Dulbecco 1999).

\subsection{PRÉD2 et sens accessoire}

Dans le cas des PRÉD2, il paraît illusoire d'unifier l'ensemble des prédications secondes en termes de 'sens accessoire'. Les adjectifs en PRÉD2 dans (17a-b), par exemple, ne sont pas supprimables :

(17) a) Il a le nez rouge / *Il a le nez

b) Il estime la solution excellente / *Il estime la solution

Leur suppression rend l'énoncé agrammatical (17a) ou pour le moins en change le sens (17b), ce qui met par ailleurs en évidence l'apport sémantique central du prédicat second. De même, certains prédicats participiaux fonctionnent comme de véritables co-verbes (cf. Herslund 2000 : 90 ) et fournissent l'apport sémantique central, ce qui peut être démontré par « le fait qu'on peut intervertir les deux verbes, le co-verbe devenant verbe principal tandis que le verbe principal, le verbe moins important, est ajouté sous forme de complément adverbial, c'est-à-dire sous forme de gérondif » (Herslund $2000: 90)(17 \mathrm{c}-\mathrm{d})$ :

(17) c) La sultane s'est levée, mettant fin à l'entretien. [= La sultane a mis fin à l'entretien en se levant]

d) La première vague reflue, emportant avec elle la plupart des gens se trouvant à la plage (TV5 11.1.05)

[En refluant, la première vague emporte avec elle...]

De plus, les constructions à PRÉD2 détachées ne se limitent pas à marquer la topicalisation à la manière des constructions disloquées. Le détachement nous paraît remplir en PRÉD2 un rôle de focalisateur fonctionnel, activant dans le cerveau les procédures d'identification de la construction PRÉD2. Divers auteurs signalent que l'élément détaché semble pouvoir remplir la fonction thématique comme rhématique.

- comme élément thématique, il se trouve le plus souvent en tête de phrase et constitue un lien avec le contexte de gauche. Il forme alors clairement une sorte de topique secondaire en reprenant directement (18a) ou indirectement (18b), un terme du contexte immédiat ou en établissant une relation avec le contexte antérieur grâce à un complément ("assurer la continuité topique", cf. Neveu $2000: 117$; cf. 21b). Le rapport prédicatif second entre l'élément détaché et son support sera caractérisé du point de vue informatif par les traits $[+\mathrm{TH} /+\mathrm{TH}]$ :

(18) a) Titre : Ministres grognons

S'estimant écartés, des membres du gouvernement critiquent la campagne «technocratique » de leur leader. (Libération 16.4.02 / 1)

b) Par-delà la présence de Nicolas Sarkozy à la Mosquée de Paris, c'est toute la question de la constitution sur l'islam en France [...], qui concerne le feu des critiques. Accédant à la demande de la Mosquée de Paris, le ministère de l'intérieur avait décidé, au mois de juin, de repousser l'élection d'une instance représentative à partir des mosquées (Le Monde 6-7.10.02 / 8)

- l'élément détaché à gauche peut aussi introduire un élément nouveau qui n'a aucun lien avec le contexte de gauche (Combettes 1998 : 62). Dans ce sens, il a un caractère rhématique (cf. Le Querler 2003), même si la prédication principale contient le 'vrai rhème' (exemple 19). Il joue 
dans cette configuration le rôle d'un 'rhème second' ${ }^{4}$ ou d'un 'rhème d'arrière-plan, tandis que son référent, qui est thématique, reprend un élément (ou plusieurs éléments) du contexte de gauche. Le rapport prédicatif second entre l'élément détaché et son support sera alors marqué par les traits $[+\mathrm{RH} /+\mathrm{TH}]$ :

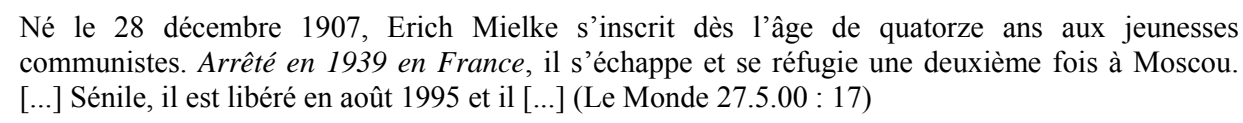

- l'élément détaché à gauche et contenant une information nouvelle peut aussi se rattacher à un support qui introduit, lui aussi, un élément nouveau (cf. Neveu 1998 : 179). A ce moment, la PRÉD2 constitue en quelque sorte un rhème complexe (20) puisque le rapport prédicatif second entre l'élément détaché et son support sera caractérisé du point de vue informatif par les traits $[+\mathrm{RH} /+\mathrm{RH}]:$

$$
\begin{aligned}
& \text { Pour faire reculer le chômage, Laguiller promet d'interdire les licenciements, tandis que Le Pen } \\
& \text { s'engage à stopper l'immigration. Morose sur le fond, l'électeur moyen attend, chaque jour qui } \\
& \text { passe, le prochain rebondissement. Les hommes et les femmes politiques ne se forcent pas pour la } \\
& \text { leur offrir. (Libération 16.4.02/15) }
\end{aligned}
$$

Certains prédicats participiaux peuvent même faire avancer l'histoire (Combettes 1998: 72 ). En conséquence, ils sont non supprimables du point de vue informatif. Dans l'exemple suivant, "cette publicité" ne peut être comprise que par l'apport de la prédication participiale :

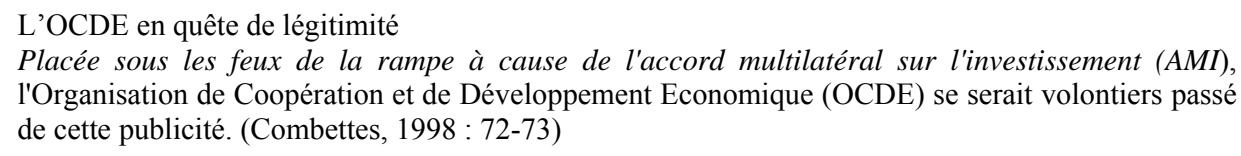

- à la place finale (22a) tout comme en position non polaire (22b), l'élément détaché semble être bien plus souvent rhématique qu'à la place initiale (cf. Combettes $1998: 67-68,83$, Neveu 1998 : 179), et marquer une forte informativité, en particulier dans des phrases à structure VS (22b). Le support peut constituer le thème (ex. 22a: $[+\mathrm{RH} /+\mathrm{TH}])$ ou le rhème $($ ex. $22 b:[+\mathrm{RH} /+\mathrm{RH}])$ de la phrase :

(22) a) Sur quoi nous allâmes boire et faire le procès du monde, tout heureux de notre entente retrouvée. (Combettes 1998: 67).

b) Là-bas se trouvait, immobile, un homme qui [...] (Combettes $1998: 67)$

Les détachements thématiques peuvent être qualifiés d'accessoires, de même que les détachements rhématiques qui ne font pas avancer l'histoire. Toutefois, dans les cas de constructions participiales mettant en évidence l'apport sémantique central, il s'agit clairement de détachements non-accessoires du point de vue informatif.

Par conséquent, fonder le rapprochement entre le détachement et la prédication seconde sur la notion d' "apport secondaire" non seulement ne donne pas de résultats probants mais ne tient pas compte en outre de l'objectif différent des opérations mises en relation : le détachement, et en son sein particulièrement la dislocation vise un but pragmatique dont l'objectif est de mettre en évidence certaines composantes du message ; la prédication seconde pour sa part est plutôt une opération de complexification d'une unité d'information.

\footnotetext{
${ }^{4}$ Pour Neveu 1998: 182-183, il s'agit cependant toujours d'un thème secondaire, parce que l'élément détaché "témoigne de son incapacité à se constituer à soi seul en objet de discours et souligne son fonctionnement en appel de support”.
} 


\section{Conclusions}

Les possibilités de chevauchement entre les propriétés du détachement et celles de la dislocation ou de la prédication seconde seront synthétisées à travers les quatre énoncés suivants :

(23) a) Marie, elle n'est pas venue.
[+DIS ; +DET ; -PRED 2]
b) Jeune, il faisait du sport.
[-DIS ; +DET ; +PRED 2]
c) De cette histoire, il se souviendra longtemps.
[-DIS ; +DET ; -PRED 2]
d) Il estime la solution excellente.
[-DIS ; -DET ; +PRED 2]

Deux observations se dégagent d'emblée : il y a recouvrement entre le détachement d'une part et la dislocation (23a) ou la prédication seconde (23b) de l'autre, mais ce recouvrement est partiel autant dans le premier que dans le second cas. Dans le cas du recouvrement DÉT/ dislocation, les exemples 23b-c soulignent qu'il n'est pas absolu ; Pour ce qui concerne le recouvrement DÉT/ PRÉD2, ce sont les énoncés 23c-d qui mettent en évidence son caractère limité.

4.1. En réalité, c'est moins le détachement que la 'non-dislocation', l'opposition avec le cas de figure envisagé sous 1.1., qui permet de caractériser la PRÉD2 dans la mesure où celle-ci n'est par définition jamais une dislocation ${ }^{5}$ : de fait, les traits +DIS et +PRÉD2 ne sont jamais simultanément présents dans 23a-d, ce qui est la conséquence logique des propriétés dégagées dans les points précédents :

- la dislocation est une extraction de PRÉD1, une rupture de la continuité phrastique originelle, une sortie du cadre propositionnel, tandis que PRÉD2 est au contraire une intégration de prédications, donc une entrée dans le cadre propositionnel;

- la dislocation implique nécessairement une trace formelle de séparation, alors que la PRÉD2 ne se marque pas par un indice formel;

- la dislocation participe nécessairement à l'organisation de la structure argumentale de PRÉD1, ce qui n'est pas le cas pour la PRÉD2 ${ }^{6}$.

4.2. Il n'y a par conséquent pas de fondement pour une caractérisation coïncidente de DÉT et de PRÉD2. De fait,

- le détachement ne caractérise pas prototypiquement l'ensemble des PRÉD2 puisque certaines PRÉD2 ne comportent pas le trait [+DET] (cf. 23d). Pour cette raison, l'appréhension de PRÉD2 d'une part et de la construction détachée de l'autre est fondamentalement différente.

Dans le premier cas (PRÉD2) le segment est vu comme l'ensemble apport + support qui se greffe sur PRÉD1 et est ainsi générateur d'une prédication complexe (PRÉD1 + PRÉD2); en conséquence, PRÉD2 est plutôt caractérisée par des marques d'intégration syntaxique (cf. la pronominalisation du thème de PRÉD2 sous la forme d'un clitique objet)

\footnotetext{
${ }^{5}$ Ce qui ne signifie pas bien sûr que les deux opérations ne peuvent pas se combiner. Ainsi, le thème d'une PRED2, qui est aussi argument dans PRED1, peut parfaitement subir une dislocation : Dulbecco \& Caddéo 2001 : 130).

${ }^{6}$ Ces observations s'opposent donc à de nombreuses grammaires qui analysent, dans des énoncés comme Pierre, il est venu, Pierre comme une apposition (Blasco-Dulbecco \& Caddéo $2001: 126$ ).
} 
Les constructions détachées sont pour leur part plutôt définies comme des segments s'appariant à un support ou à un référent dans PRÉD1 ("Dépendance référentielle de la CD envers le groupe référentiel (le référent) dans PRÉD1" (Combettes 1998 : 13-15; 27)). Elles sont par conséquent identifiées - c'est une de leurs propriétés de base - par leur disjonction formelle, matérialisée par la discontinuité, qui s'exprime par une liberté de position et des marques prosodiques ou diacritiques.

- la construction détachée ne remplit pas non plus dans le contexte de PRÉD2 le rôle d'une structure marquée par rapport à une séquence sans détachement qui remplirait le rôle d'une structure non marquée :

(24) a) De son père, il refuse toute aide.

b) Il refuse toute aide de son père.

c) Mécontent, l'homme a quitté la salle.

d) L'homme, mécontent, a quitté la salle.

d') L'homme mécontent a quitté la salle.

On peut en effet considérer la construction détachée $24 \mathrm{a}$ comme la forme marquée par rapport à une séquence non détachée et non marquée $24 \mathrm{~b}$. Cette opposition est toutefois difficile à maintenir dans le cas de $(24 c-d)$ où l'adjectif doit par définition être détaché dans les deux énoncés pour être considéré comme une PRÉD2 (cf. 24d-d'). En conséquence, dans ces tours, le détachement ne peut être considéré comme une indication de marquage informationnel, comme c'est le cas dans les exemples 24a-b. Dans les prédications secondes, le détachement joue donc un autre rôle : c'est fondamentalement un indice d'extraprédicativité : il souligne que l'élément en question ne fonctionne, ni comme argument du prédicat central, ni comme déterminant d'un de ces arguments. C'est un rôle largement rempli dans de nombreuses constructions à prédication seconde $\left(25 \mathrm{a}><25 \mathrm{~b} ; 1 \mathrm{~d}-\mathrm{d}^{\prime}><1 \mathrm{~d}^{\prime \prime}\right)$ :

(25) a) Les hommes, qui étaient malades, ont été déportés

b) Les hommes qui étaient malades ont été déportés.

(1) d) Regardant à sa gauche, il vit le facteur qui sortait de sa maison.

d') D'autant que la loi Evin, prohibant déjà le tabagisme dans certains lieux publics, n'est guère appliquée (Le Figaro 10.12.03 / 12).

d') Pour certains, un texte interdisant de fumer n'est toutefois pas inconcevable à terme (Le Figaro 10.12.03/12).

Le détachement joue donc un rôle de focalisation fonctionnelle, en activant les procédures d'identification de la construction PRÉD2. Même dans le cas de constructions marquées au sein de PRÉD2 par le non-détachement, les 'attributs de l'objet' par exemple (26a), elles présentent très fréquemment des emplois avec pronominalisation du support objet (26b). L'objet clitique se positionnant devant le verbe, elles rétablissent de la sorte une structure détachée - mais marquée dans la mesure où c'est le support qui est détaché - et distinguent formellement l'interprétation prédicative seconde d'une interprétation déterminative (26b) :

(26) a) Il a acheté sa voiture neuve

b) Il l'a enfin achetée, sa voiture neuve / Il l'a achetée neuve, sa voiture

Dans le cas d'une grammaticalisation (ou spécification syntaxique) du lien de prédication seconde toutefois, le détachement ne s'impose plus comme discriminant fonctionnel (26c-d) :

c) Il y a une place de libre $><$ Il a occupé une place libre 
d) Il considère ce groupe armé comme particulièrement dangereux $><$ ce groupe armé particulièrement dangereux a revendiqué l'enlèvement de Jean.

Notre étude se donnait pour objectif d'évaluer si le détachement constituait une propriété basique de la prédication seconde. L'analyse systématique des propriétés de ces deux notions nous amène à conclure qu'on ne peut assimiler sans plus le détachement, que ce soit dans une saisie étroite ou une appréhension plus large, à la prédication seconde, ni que cette dernière puisse être caractérisée basiquement par la première : Le détachement en tant que notion "ne saurait donc marquer une identité fonctionnelle" (Neveu 2003 : 9). Un tel constat n'implique évidemment nullement que le détachement ne remplirait aucun rôle dans les PRÉD2 mais la caractérisation de celui-ci dépasse les objectifs de la présente contribution.

\section{Bibliographie sommaire}

Blanche-Benveniste, Claire \& Caddéo, Sandrine (2000), "Préliminaires à une étude de l'apposition dans la langue parlée". Langue française 125, 60-70.

Blasco-Dulbecco, Mylène (1999), Les dislocations en français contemporain. Paris, Honoré Champion.

Blasco-Dulbecco, Mylène \& Caddéo, Sandrine (2001), “Apposition et dislocation: la séquence pronom+lexique+clitique". Recherches sur le français parlé 16, 125-149.

Cadiot , Pierre \& Furukawa, Naoyo (éds) (2000), “La prédication seconde”. Langue française, 127.

Combettes, Bernard (1998), Les constructions détachées en français. Paris, Ophrys.

Dupont, Norbert (1985), Linguistique du détachement en français. Berne, Éditions Peter Lang SA.

Forsgren, M. (2000), “Apposition, attribut, épithète : même combat prédicatif ?" Langue française 125: 3045.Forsgren, Mats (1986) "Élements pour une typologie de l'apposition en linguistique française. "Actes du XVIIIe Congrès International de Linguistique et de Philologie Romanes, Dieter Kremer (éd.), Max Niemeyer Verlag, Tübingen. Pp. 597-612.

Fradin, Bernard (1988), “Approche des constructions à détachement : la reprise interne”. Langue francaise, 78, 26-56.

Fradin, Bernard (1990), “Approche des constructions à détachement : inventaire". Revue Romane 25 : 1, 3-34.

Furukawa, Naoyo (2003), "Les éléments initiaux détachés et la thématisation”. Cahiers de praxématique 40, 127-147.

Grevisse, Maurice $\left(1975^{10}\right)$, Le bon usage. Grammaire française avec des remarques sur la langue française d'aujourd'hui. Gembloux, Duculot.

Havu, Eva (2004), "L’interprétation des constructions participiales appositives". La Linguistique 40, 65-82.

Havu, Eva \& Pierrard, Michel (2004), "La prédication seconde de type adjectival : une structure attributive sans verbe copule ?" Javier Suso López \& Rodrigo López Carrillo (éds.) Le français face aux défis actuels. Histoire, langue et culture. Granada, APFUE-GILEC, 417-431.

Herslund, Michael (2000), “Le participe présent comme co-verbe”. Langue française 127, 86-94

Lambrecht, Knud (2001), "Dislocation”.Haspelmatth M. et al. (éds) Language Typology and Language Universals. Berlin-New York, Walter de Gruyter.

Le Querler, Nicole (2003) “Le nominativus pendens en français”. Cahiers de praxématique 40, 149-165.

Mertens, Piet (1990), “Intonation”. Blanche-Benveniste, Claire (éd.) Le français parlé. Études grammaticales. Paris, CNRS, 159-176.

Morel, Mary-Annick (1992), “Intonation et thématisation”.. L'Information grammaticale 54, 26-36.

Neveu, Franck (1998), Études sur l'apposition. Paris, Honoré Champion. 


\section{InforGram}

Neveu, Franck (2000), "Quelle syntaxe pour l'apposition ? Les types d'appariement des appositions frontales et la continuité référentielle". Langue française 125, 106-124.

Neveu, Franck (2003), “Détachement, adjonction, discontinuité, incidence...”. Cahiers de praxématique 40, 7-19.

Prévost, Sophie (2003), "Détachement et topicalisation : des niveaux d'analyse différents". Cahiers de praxématique 40, 97-125.

Willems, Dominique (1998), "La mer, c'est beau. Le sujet dans certaines structures non canoniques". Forsgren, M., Jonasson, K. et H. Kronning (éds) Prédication, assertion, information. Uppsala, Acta Universitatis Upsaliensis, 595603. 